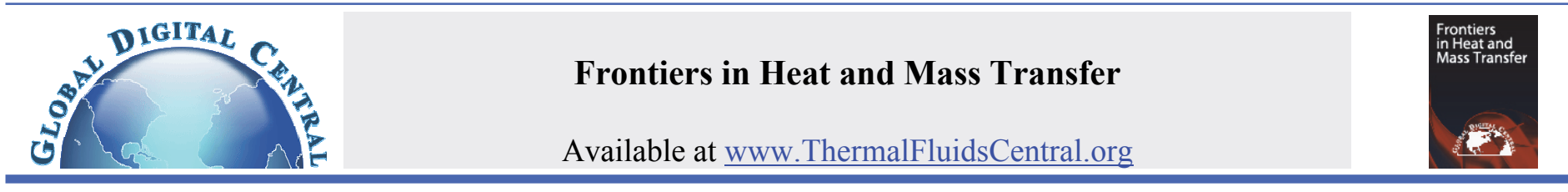

\title{
EFFECTS OF VARIABLE VISCOSITY AND VARIABLE THERMAL CONDUCTIVITY ON HYDROMAGNETIC DUSTY FLUID FLOW DUE TO A ROTATING DISK
}

\author{
Jadav Konch ${ }^{*}$ and G. C. Hazarika \\ Department of Mathematics, Dibrugarh University, Dibrugarh-786004, Assam, India
}

\begin{abstract}
This paper investigates momentum, heat and mass transfer characteristics of a hydromagnetic Newtonian dusty fluid flow due to a rotating disk with radiation and viscous dissipation. The main objective of this paper is to study effects of temperature dependent viscosity and thermal conductivity on flow, temperature and species concentration. Radiation and viscous dissipation effects are also taken into account. Saffman model for dusty fluid is considered for the problem. The partial differential equations governing the flow are converted into ordinary differential equations employing similarity transformations. The resulting highly nonlinear coupled ordinary differential equations are solved numerically using shooting technique with fourth order Runge-Kutta integration scheme. The influence of variable fluid viscosity, variable thermal conductivity, fluid particle interaction, magnetic interaction and radiation parameters as well as Eckert number are presented graphically and discussed for velocity, temperature and species concentration profiles. Numerical values of the wall shear stress, rate of heat transfer (in terms of Nusselt number) and rate of mass transfer (in terms of Sherwood number) are obtained, analyzed and presented in tabular form.
\end{abstract}

Keywords: Dusty fluid, variable viscosity, variable thermal conductivity, radiation, rotating disk, shooting method.

\section{INTRODUCTION}

The study of a two-phase gas-particle flow has a wide range of applications in power plant piping, combustion, petroleum transport, waste water treatment, corrosive particles in engine oil flow and formation of raindrops. Its relevance is also seen in the field of smoke emission from vehicles, agriculture and food technologies. Motivated by the applications of a two-phase flow, Saffman (1962) has initiated and formulated the governing equations for the flow of dusty fluid and discussed the stability of a laminar flow of a dusty gas. In this model, he considered that the dust particles were uniformly distributed. Michael and Miller (1966) discussed the motion of dusty gas occupying the semi infinite space above a rigid plane boundary. Nayfeh (1966) studied oscillating dusty flow through a rigid pipe. Gupta and Gupta (1976) discussed the flow of a gas containing solid particles in a channel with arbitrary time varying pressure. The flow of a dusty fluid in the boundary layer over a semi-infinite flat plate was analyzed by Datta and Mishra (1982). Ramamurthy (1990) investigated the effects of free convection on the Stokes problem for the flow of dusty fluid in an infinite vertical plate. Attia (2006) analyzed unsteady MHD Couette flow and heat transfer of dusty fluid with variable physical properties. Palani and Kim (2010) obtained the approximate solution for the flow of a dusty-gas along a semi-infinite vertical cylinder. Gireesha et al. $(2011 ; 2012$; 2013) discussed interesting results on the flow of dusty fluid due to linear and exponential stretching of porous and nonporous sheet with various effects like radiation, source/sink parameter, viscous dissipation etc. Manjunatha (2015) studied the effect of radiation on MHD flow and heat transfer of dusty fluid over a stretching cylinder in a porous medium.

The study of dusty viscous fluid flow under the influence of various physical conditions has been carried out by several researchers or authors. This interest stems from the fact that the flow and heat transfer of magnetohydrodynamic boundary layer flow finds its applications in wide range of science and technology like MHD power generation, purification of crude oil, cooling of nuclear reactors, centrifugal separation of matter from fluid and in several astrophysical situations. The theory of rotating fluid is highly important due to its occurrence in various natural phenomena and for its applications in various technological situations. The broad subjects of oceanography, meteorology, atmospheric sciences, and limnology all contain some important and essential features of rotating fluid.

Further, the fluid flow problems of rotating fluid have attracted many scholars and there appeared a number of studies in literature viz. Tiwari and Kamal Singh (1983) analyzed viscous incompressible hydromagnetic boundary layer flow of dusty fluid over a semi-infinite plate. Prasada Rao and Krishna (1981) investigated the Hall Effect on unsteady hydromagnetic flow. The effects of Hall current on unsteady MHD flow past a rotating disk was studied by Kanch and Jana (1999). Debnath et al. (1979) studied the Hall effects on the unsteady hydromagnetic flow of a rotating fluid system over a porous plate.

Turkyilmazoglu $(2009 ; 2010)$ studied the steady laminar flow of viscous, incompressible, electrically-conducting fluid over a rotating disk in the presence of a transverse magnetic field. The problem of heat transfer from a rotating disk maintained at a constant temperature for various values of Prandtl number in steady state was considered by Millsaps and Pohlhausen (1952). Heat transfer characteristic of MHD steady flow of a viscous incompressible electrically conducting fluid past a rotating disk with Hall current, viscous dissipation and Ohmic heating were studied by Sibanda and Makinde (2010). They found that magnetic field opposed fluid motion due to the Lorentz force generated by the magnetic field.

It is interesting that in the above flow problems generated by a rotating disk, fluids are considered in pure form. But, it is well known

* Corresponding Author Email: jadavkonch@gmail.com 
that air and water, by nature, contain impurities like dust particles and foreign bodies. In fact, the problem of two-phase flows in which solid particles are distributed in clean fluid has several practical applications such as the environmental pollution, sedimentation, centrifugal separation of particles, blood rheology, purification of crude oil and physiological flows.

Moreover, from the above studies, it is also seen that most of the investigators restricted their investigations by assuming viscosity and thermal conductivity of the fluid as constant. However, it was known from the work of Herwig and Wicken (1986) that these properties may change with temperature. A theoretical investigation of the effects of temperature dependent viscosity on forced convection over a flat plate in porous medium has been presented by Ling and Dybbs (1987). So, a more accurate prediction for the flow, heat transfer and mass transfer can be achieved by taking into account the variation of such properties with temperature. Hazarika and Konch (2016) and Konch and Hazarika (2016) have studied the influence of varying viscosity and thermal conductivity on convective heat and mass transfer in the flow of dusty fluid with radiation and viscous dissipation.

Motivated by the above-mentioned investigations and applications, the aim of this work is to investigate effects of temperature dependent fluid viscosity and thermal conductivity on the hydromagnetic boundary layer flow of a dusty fluid due to a rotating disk with radiation and viscous dissipation. Shooting technique with fourth order Runge-Kutta integration scheme is employed to obtain the solution of the boundary value problem.

\section{MATHEMATICAL FORMULATION}

Consider the problem of steady, hydromagnetic axially symmetric flow of an electrically conducting incompressible dusty fluid with viscous dissipation and radiation. The flow is generated due to a rotating disk. It is assumed that fluid is extent infinitely in the positive z-direction. Let $(r, \phi, z)$ be the set of cylindrical polar coordinates and let the disk be rotates with constant angular velocity $\vec{\Omega}\left(0,0, \Omega_{0}\right)$ and be placed at $z=0$ as indicated in Fig. 1.

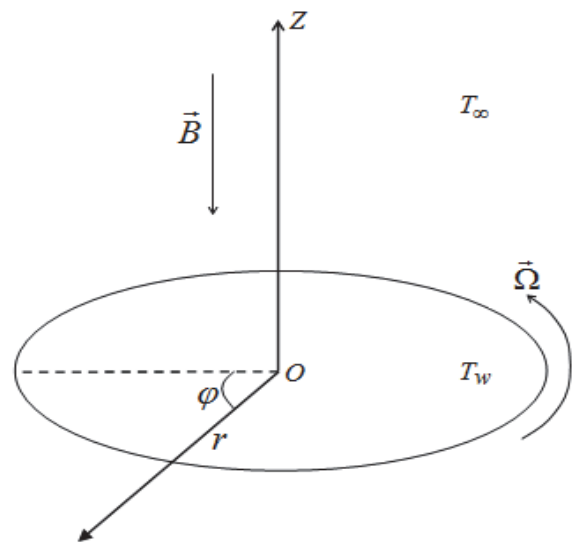

Fig. 1 Schematic diagram of the problem

The fluid and dust phase velocity components are $(u, v, w)$ and $\left(u_{p}, v_{p}, w_{p}\right)$ respectively in the directions of $(r, \varphi, z)$. The surface of the rotating disk is maintained at uniform temperature $T_{w}$. The free stream (far away from the wall) is kept at constant temperature $T_{\infty}$ and at constant pressure $P_{\infty}$. An external uniform magnetic field of strength $\vec{B}\left(0,0, B_{0}\right)$ is applied perpendicular to the surface of the disk.
The analysis is based on the following assumptions:

- Fluid properties are assumed as constant except the fluid viscosity and thermal conductivity.

- Dust particles are assumed as electrically non-conducting, spherical in shape having same radius and mass, and undeformable.

- The number density of dust particles is taken as constant throughout the flow.

- Magnetic Reynolds number is assumed to be so small therefore the induced magnetic field is negligible (Sutton (1965)).

Under these assumptions together with Devi and Devi (2011), Khidir (2013), Boussinesq approximations and following Saffman (1962) model of dusty gas the governing equations are:

For the fluid phase:

$$
\begin{aligned}
& \frac{\partial u}{\partial r}+\frac{u}{r}+\frac{\partial w}{\partial z}=0 \\
& \rho\left(u \frac{\partial u}{\partial r}-\frac{v^{2}}{r}+w \frac{\partial u}{\partial z}\right)=-\frac{\partial p}{\partial r}+\frac{\partial}{\partial r}\left(\mu \frac{\partial u}{\partial r}\right)+\frac{\partial}{\partial r}\left(\mu \frac{u}{r}\right) \\
& +\frac{\partial}{\partial z}\left(\mu \frac{\partial u}{\partial z}\right)-\sigma B_{0}^{2} u+K N\left(u_{p}-u\right) \\
& \rho\left(u \frac{\partial v}{\partial r}+\frac{u v}{r}+w \frac{\partial v}{\partial z}\right)=\frac{\partial}{\partial r}\left(\mu \frac{\partial v}{\partial r}\right)+\frac{\partial}{\partial r}\left(\mu \frac{v}{r}\right)+\frac{\partial}{\partial z}\left(\mu \frac{\partial v}{\partial z}\right) \\
& -\sigma B_{0}^{2} v+K N\left(v_{p}-v\right) \\
& \rho\left(u \frac{\partial w}{\partial r}+w \frac{\partial w}{\partial z}\right)=-\frac{\partial p}{\partial z}+\frac{\partial}{\partial r}\left(\mu \frac{\partial w}{\partial r}\right)+\frac{1}{r} \frac{\partial}{\partial r}(\mu w) \\
& +\frac{\partial}{\partial z}\left(\mu \frac{\partial w}{\partial z}\right)+K N\left(w_{p}-w\right) \\
& \rho c_{p}\left(u \frac{\partial T}{\partial r}+w \frac{\partial T}{\partial z}\right)=\frac{\partial}{\partial r}\left(\lambda \frac{\partial T}{\partial r}\right)+\frac{\lambda}{r} \frac{\partial T}{\partial r}+\frac{\partial}{\partial z}\left(\lambda \frac{\partial T}{\partial z}\right)-\frac{\partial q_{r}}{\partial z} \\
& +\mu\left[\left(\frac{\partial u}{\partial z}\right)^{2}+\left(\frac{\partial v}{\partial z}\right)^{2}\right]+\frac{N c_{p}}{\tau_{T}}\left(T_{p}-T\right) \\
& u \frac{\partial C}{\partial r}+w \frac{\partial C}{\partial z}=\frac{1}{r} \frac{\partial}{\partial r}\left(r D_{m} \frac{\partial C}{\partial r}\right)+\frac{\partial}{\partial z}\left(D_{m} \frac{\partial C}{\partial z}\right) \\
& +\frac{m N}{\rho \tau_{c}}\left(C_{p}-C\right)
\end{aligned}
$$

For the dust phase:

$$
\begin{aligned}
& \frac{1}{r} \frac{\partial}{\partial r}\left(r \rho_{p} u_{p}\right)+\frac{\partial}{\partial z}\left(\rho_{p} w_{p}\right)=0 \\
& m\left(u_{p} \frac{\partial u_{p}}{\partial r}-\frac{v_{p}^{2}}{r}+w_{p} \frac{\partial u_{p}}{\partial z}\right)=-K\left(u_{p}-u\right) \\
& m\left(u_{p} \frac{\partial v_{p}}{\partial r}+\frac{u_{p} v_{p}}{r}+w_{p} \frac{\partial v_{p}}{\partial z}\right)=-K\left(v_{p}-v\right) \\
& m\left(u_{p} \frac{\partial w_{p}}{\partial r}+w_{p} \frac{\partial w_{p}}{\partial z}\right)=-K\left(w_{p}-w\right)
\end{aligned}
$$


$N c_{m}\left(u_{p} \frac{\partial T_{p}}{\partial r}+w_{p} \frac{\partial T_{p}}{\partial z}\right)=-\frac{N c_{p}}{\tau_{T}}\left(T_{p}-T\right)$

$u_{p} \frac{\partial C_{p}}{\partial r}+w_{p} \frac{\partial C_{p}}{\partial z}=-\frac{m N}{\rho \tau_{c}}\left(C_{p}-C\right)$

Appropriate boundary conditions for the problem are:

$\left.\begin{array}{l}\text { At } z=0: u=0, v=\Omega_{0} r, w=0, T=T_{w}, C=C_{w}, \\ \text { As } z \rightarrow \infty: u=0, v \rightarrow 0, u_{p} \rightarrow 0, v_{p} \rightarrow 0, w_{p} \rightarrow w, \\ T \rightarrow T_{\infty}, T_{p} \rightarrow T_{\infty}, \rho_{p} \rightarrow \omega \rho, C \rightarrow C_{\infty}, C_{p} \rightarrow C_{\infty}\end{array}\right\}$

Using Rosseland approximation for radiation we can write radiative heat flux as (Necati (1973))

$$
q_{r}=-\frac{4 \sigma *}{3 k *} \frac{\partial T^{4}}{\partial z},
$$

where $\sigma^{*}$ and $k^{*}$ are the Stefan-Boltzmann constant and mean absorption coefficient, respectively.

It is assumed that temperature difference within the flow such that the term $T^{4}$ can be expressed as a linear function of temperature. This is accomplished by expanding $T^{4}$ in a Taylor series about $T_{\infty}$ and neglecting the second and higher order terms, we get

$$
T^{4} \cong 4 T_{\infty}^{3} T-3 T_{\infty}^{4}
$$

In order to obtain ordinary differential equations, we introduce the following similarity transformations:

$$
\left.\begin{array}{l}
\eta=\left(\frac{\Omega_{0}}{v_{\infty}}\right)^{1 / 2} z, u=\Omega_{0} r f(\eta), v=\Omega_{0} r g(\eta), \\
w=\left(\Omega_{0} v_{\infty}\right)^{1 / 2} h(\eta), p-p_{\infty}=2 \mu_{\infty} \Omega_{0} P(\eta), u_{p}=\Omega_{0} r F(\eta), \\
v_{p}=\Omega_{0} r g G(\eta), w_{p}=\left(\Omega_{0} v_{\infty}\right)^{1 / 2} \mathcal{H}(\eta), \theta=\frac{T-T_{\infty}}{T_{w}-T_{\infty}}, \\
\theta_{p}=\frac{T_{p}-T_{\infty}}{T_{w}-T_{\infty}}, \phi=\frac{C-C_{\infty}}{C_{w}-C_{\infty}}, \phi_{p}=\frac{C_{p}-C_{\infty}}{C_{w}-C_{\infty}}, \rho_{r}=\mathcal{H}(\eta)
\end{array}\right\}
$$

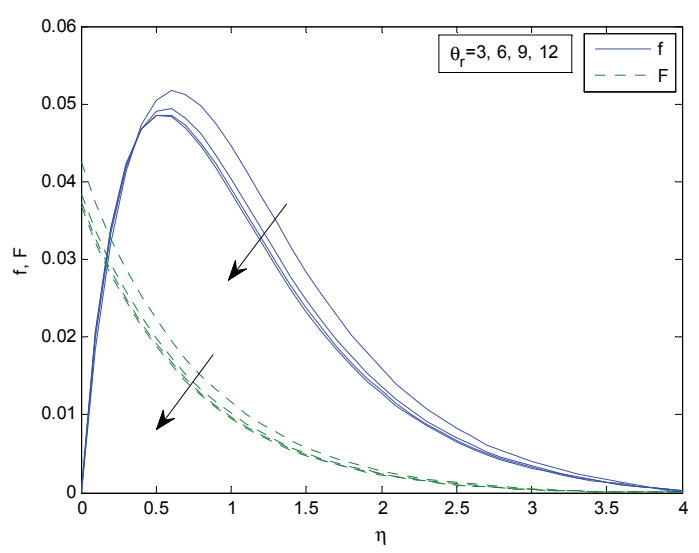

Fig. 2 Radial velocity profile for different $\theta_{r}$

The viscosity of the fluid is assumed to be an inverse linear function of temperature, and it can be expressed as following Lai and Kulacki (1991):
$\frac{1}{\mu}=\frac{1}{\mu_{\infty}}\left[1+\delta\left(T-T_{\infty}\right)\right] \quad$ or, $\frac{1}{\mu}=\alpha\left(T-T_{r}\right)$,

where $\alpha=\frac{\delta}{\mu_{\infty}}$ and $T_{r}=T_{\infty}-\frac{1}{\delta}$.

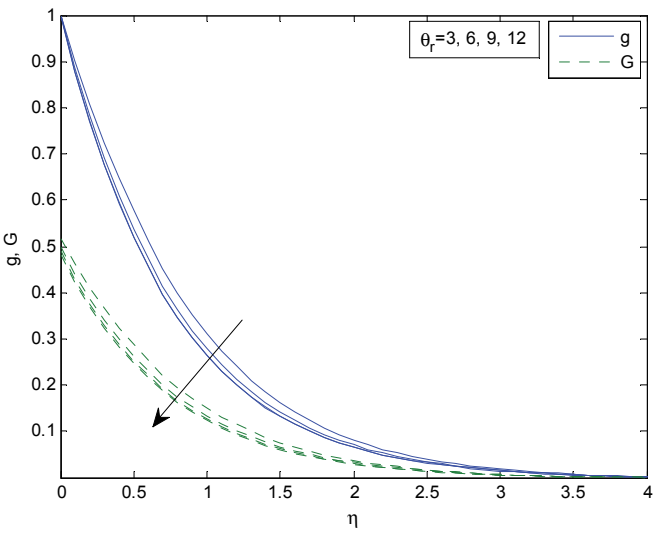

Fig. 3 Tangential velocity profile for different $\theta_{r}$

Moreover, the thermal conductivity of the fluid varies with temperature. Following Choudhury and Hazarika (2013), we assumed thermal conductivity of the fluid as:

$\frac{1}{\lambda}=\frac{1}{\lambda_{\infty}}\left[1+\xi\left(T-T_{\infty}\right)\right]$ or, $\frac{1}{\lambda}=\zeta\left(T-T_{C}\right)$,

where $\zeta=\frac{\xi}{\lambda_{\infty}}$ and $T_{c}=T_{\infty}-\frac{1}{\xi}$.

Here $\alpha, \xi, \zeta, T_{r}$ and $T_{c}$ are constants and their values depend on the reference state and thermal properties of the fluid i.e., $v$ (kinematic viscosity) and $\lambda$ (thermal conductivity).

Let us introduce two dimensionless parameters:

$\theta_{r}=\frac{T_{r}-T_{\infty}}{T_{w}-T_{\infty}}$ the dimensionless reference temperature corresponding to viscosity called the viscosity variation parameter, and $\theta_{c}=\frac{T_{c}-T_{\infty}}{T_{w}-T_{\infty}}$ the dimensionless reference temperature corresponding to thermal conductivity called the thermal conductivity variation parameter.

It is also important to note that $\theta_{r}$ and $\theta_{c}$ are negative for liquids and positive for gases (Kuppalapalle et al. (2013)).

Using these two parameters in equations (17) and (18) we get dynamic viscosity and thermal conductivity as:

$$
\mu=-\frac{\mu_{\infty} \theta_{r}}{\theta-\theta_{r}} \text { and } \lambda=-\frac{\lambda_{\infty} \theta_{c}}{\theta-\theta_{c}} .
$$

Substituting equations (14)-(19) into equations (1)-(13), we get the following nonlinear ordinary differential equations:

$f=-\frac{1}{2} h^{\prime}$

$\frac{\theta_{r}}{\theta-\theta_{r}} h^{\prime \prime \prime}-\frac{\theta_{r}}{\left(\theta-\theta_{r}\right)^{2}} \theta^{\prime} h^{\prime \prime}+h h^{\prime \prime}+\frac{1}{2} h^{\prime 2}+M h^{\prime}$ 
$+2 l \beta_{v} \mathcal{H}\left(F+\frac{1}{2} h^{\prime}\right)+2 g^{2}=0$

$\frac{\theta_{r}}{\theta-\theta_{r}} g^{\prime \prime}-\frac{\theta_{r}}{\left(\theta-\theta_{r}\right)^{2}} \theta^{\prime} g^{\prime}+h g^{\prime}+M g^{\prime}-h^{\prime} g-l \beta_{v} \mathcal{H}(G-g)=0$

$\left(\frac{\theta_{c}}{\theta-\theta_{c}}-\frac{4}{3 R_{d}}\right) \theta^{\prime \prime}-\frac{\theta_{c}}{\left(\theta-\theta_{c}\right)^{2}} \theta^{2}+\operatorname{Pr} h \theta^{\prime}+E c \operatorname{Pr} \frac{\theta_{r}}{\theta-\theta_{r}}\left(\frac{1}{4} h^{\prime \prime 2}+g^{\prime 2}\right)$

$-\operatorname{Pr} \beta_{T} a_{1}\left(\theta_{p}-\theta\right)=0$

$\frac{\theta_{r}}{\theta-\theta_{r}} \phi^{\prime \prime}-\frac{\theta_{r}}{\left(\theta-\theta_{r}\right)^{2}} \theta^{\prime} \phi^{\prime}+S c h \phi^{\prime}-S c l \beta_{c} \mathcal{H}\left(\phi_{p}-\phi\right)=0$

$H \mathcal{H}^{\prime}+H^{\prime} \mathcal{H}+2 F \mathcal{H}=0$

$H F^{\prime}+F^{2}+\beta_{v}\left(F+\frac{1}{2} h^{\prime}\right)-G^{2}=0$

$H G^{\prime}+2 F G+\beta_{v}(G-g)=0$

$H H^{\prime}+\beta_{v}(H-h)=0$

$H \theta_{p}^{\prime}+\gamma \beta_{T}\left(\theta_{p}-\theta\right)=0$

$H \phi_{p}^{\prime}+l \beta_{c} \mathcal{H}\left(\phi_{p}-\phi\right)=0$

Boundary conditions are reduced to:

$\left.\begin{array}{c}\text { At } \eta=0: \quad f=0, h=0, h^{\prime}=0, g=1, \theta=1, \phi=1 \\ \text { As } \eta \rightarrow \infty: f=0, h=h_{w}, h^{\prime}=0, g=0, F=0, G=0, \\ \mathcal{H}=\omega, \theta=0, \theta_{p}=0, \phi=0, \phi_{p}=0 .\end{array}\right\}$

where the dimensionless parameters are defined as follows:

$M=\frac{\sigma B_{0}^{2}}{\Omega_{0} \rho}$ is the magnetic parameter; $\operatorname{Re}=\frac{\Omega_{0} r^{2}}{v_{\infty}}$ is the Reynolds number; $R_{d}=\frac{\lambda_{\infty} k^{*}}{4 \sigma^{*} T_{\infty}^{3}}$ is the radiation parameter; $\operatorname{Pr}=\frac{\mu_{\infty} c_{p}}{\lambda_{\infty}}$ is the Prandtl number; $S c=v / D_{m}$ is the Schmidt number. $\beta_{v}=1 / \tau_{v} \Omega_{0}$, $\beta_{T}=1 / \tau_{T} \Omega_{0}$ and $\beta_{c}=1 / \tau_{c} \Omega_{0}$ are the fluid particle interaction parameters for velocity, temperature and concentration, respectively; $\tau_{v}=m / K$ is the velocity equilibrium time; $l=m N / \rho_{p}$ is the mass concentration; $\rho_{r}=\rho_{p} / \rho$ is the relative density; $\gamma=c_{p} / c_{m}$ is the ratio of specific heat; $a_{1}=N / \rho$ is a constant and a prime $\left({ }^{\prime}\right)$ denotes derivative with respect to similarity variable $\eta$.

\subsection{Skin friction Coefficient, Nusselt Number and Sherwood Number}

Skin friction coefficient in the radial $\left(C_{f r}\right)$ and tangential $\left(C_{f t}\right)$ directions, Nusselt number $(\mathrm{Nu})$ and Sherwood number $(\mathrm{Sh})$ are the parameters of physical and engineering interest for the present problem, which physically indicates the wall shear stress, rate of heat transfer and rate of mass transfer, respectively.

Skin friction coefficient in the radial and tangential direction are defined as
$C_{f r}=\left[\frac{\tau_{r}}{\rho u^{2} / 2}\right]_{z=0}$, where $\tau_{r}=\left[\mu\left(\frac{\partial u}{\partial z}+\frac{\partial w}{\partial r}\right)\right]$ is the radial

shear stress; and

$C_{f t}=\left[\frac{\tau_{t}}{\rho v^{2} / 2}\right]_{z=0}$, where $\tau_{t}=\left[\mu \frac{\partial v}{\partial z}+\frac{1}{r} \frac{\partial w}{\partial \varphi}\right]$ is the tangential shear stress, respectively.

Using the non-dimensional variables we get the skin friction coefficients as

$C_{f r} \mathrm{Re}^{1 / 2}=\frac{\theta_{r}}{1-\theta_{r}} h^{\prime \prime}(0)$ and $\quad \frac{1}{2} C_{f t} \mathrm{Re}^{1 / 2}=-\frac{\theta_{r}}{1-\theta_{r}} g^{\prime}(0)$.

The Nusselt number is defined as

$N u=\frac{x q_{w}}{\lambda_{\infty}\left(T_{w}-T_{\infty}\right)}$, where $\left.q_{w}=-\lambda \frac{\partial T}{\partial z}\right)_{z=0}$ is the heat transfer

from surface of the disk.

Using the non-dimensional variables we get

$N u / \mathrm{Re}^{1 / 2}=\frac{\theta_{c}}{1-\theta_{c}} \theta^{\prime}(0)$.

The Sherwood number which is defined as

$S h=\frac{x m_{w}}{D m_{\infty}\left(C_{w}-C_{\infty}\right)}$, where $\left.m_{w}=-D_{m} \frac{\partial C}{\partial z}\right)_{z=0}$ is the mass flux

at the surface and $D m_{\infty}$ is the diffusion constant at the free stream.

Using the non-dimensional variables we get

$S h / \mathrm{Re}^{1 / 2}=\frac{\theta_{r}}{1-\theta_{r}} \phi^{\prime}(0)$.

\section{METHOD OF SOLUTION}

The equations (20)-(30) governing the flow subject to boundary conditions represented by equation (31) are solved numerically using shooting technique with the fourth order Runge-Kutta integration scheme. In this method, all higher order nonlinear ordinary differential equations are converted into a system of linear differential equations of first order and then they are transformed into initial value problem applying shooting technique (Mallikarjuna et al. (2016)). Numerical values are computed by developing suitable codes in MATLAB for the method. From the process of numerical computation, the velocity, temperature and concentration profiles for both the fluid and dust phases are plotted for various flow governing parameters. Further, the skin friction coefficients, Nusselt number and Sherwood number are sorted out and their numerical values are presented in tabular form.

\section{RESULTS AND DISCUSSION}

In order to analyze the problem physically, a representative set of numerical results of velocity components, temperature and concentration profiles are shown graphically in Figs. 2-23. The coefficients of skin friction in the radial and tangential directions, Nusselt number and Sherwood number are also calculated and are presented in tabular form. The results are given for different values of the various dimensionless parameters governing the flow, viz., viscosity variation parameter $\left(\theta_{r}\right)$, thermal conductivity variation parameter $\left(\theta_{c}\right)$, fluid particle interaction parameters $\left(\beta_{v}, \beta_{T}, \beta_{c}\right)$, magnetic parameter $(M)$, radiation parameter $\left(R_{d}\right)$ and Eckert number $(E c)$. Numerical values of the parameters used for simulation 
are: $M=1, \operatorname{Pr}=0.71, \beta_{v}=\beta_{T}=\beta_{c}=2, E c=0.05, R_{d}=5, S c=0.22, \theta_{r}=5$ and $\theta_{c}=3$ unless otherwise stated.

Figures 2-6 demonstrate the influence of viscosity variation parameter $\left(\theta_{r}\right)$ on velocity, temperature and concentration profiles of fluid and dust phase. It is seen from Figs. 2-3 that an increase in the strength of $\theta_{r}$ leads to retard the radial and tangential velocity of both the phases. Physically as the values of viscosity variation parameter $\theta_{r}$ increases, the resistance to the relative motion of the different layers of a fluid increase due to the increase of viscous force and as a result velocity of the fluid decreases. But Fig. 4 shows a back flow in the axial direction. It is observed from Fig. 5 that temperature of the fluid and dust phase increases when viscosity variation parameter $\theta_{r}$ is increased. Physically an enhancement of $\theta_{r}$ leads to increase flow resistive viscous force and therefore to overcome this force and maintain the flow, the fluid has to do extra work. That energy transformed into thermal energy as a result temperature of the fluid increases. At the same time concentration of both the fluid and dust phases decrease for increasing $\theta_{r}$ (Fig. 6).

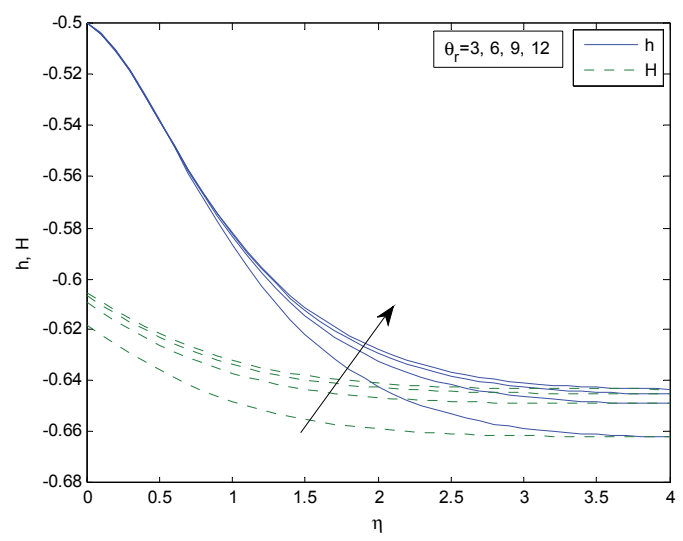

Fig. 4 Axial velocity profile for different $\theta_{r}$

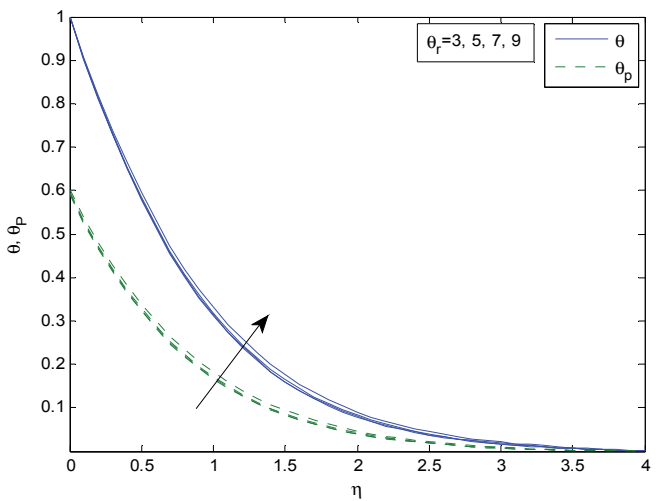

Fig. 5 Temperature profile for different $\theta_{r}$

From Fig. 7, we have found that both the fluid and dust phase temperature depreciate with an increase in the strength of the thermal conductivity variation parameter $\theta_{C}$. The reason behind is that thermal conductivity is an inverse linear function of temperature, hence the result.

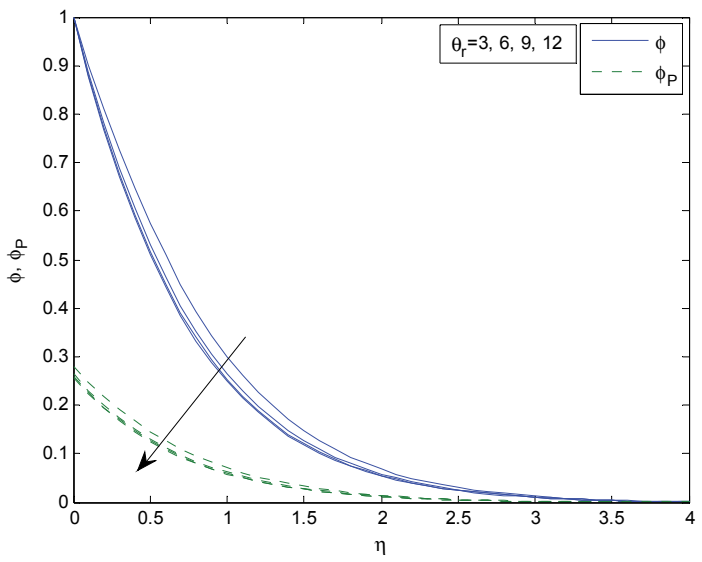

Fig. 6 Concentration profile for different $\theta_{r}$

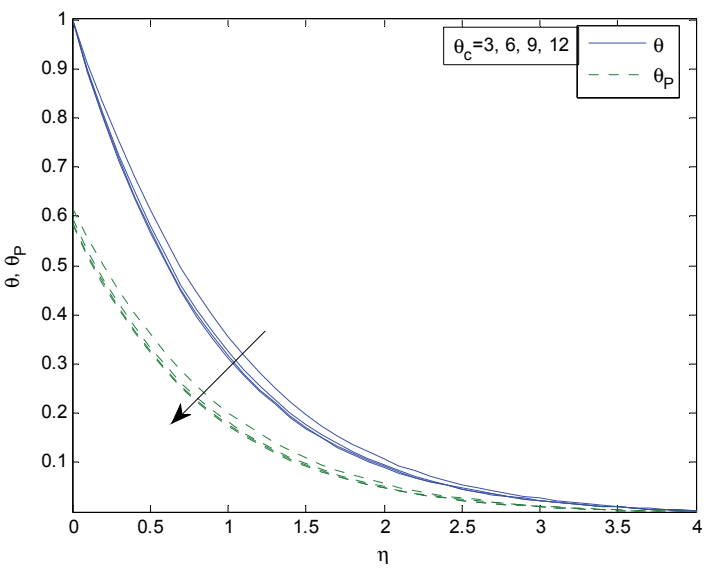

Fig. 7 Temperature profile for different $\theta_{c}$

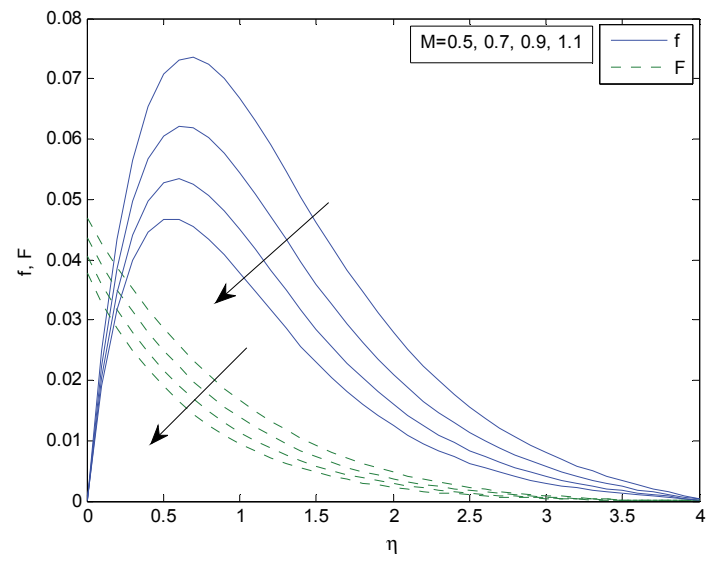

Fig. 8 Radial velocity profile for different $M$

Figures 8-12 illustrate the effect of magnetic field on velocity, temperature and concentration profiles through the magnetic parameter $(M)$. As the increasing values of magnetic parameter $M$, radial and tangential components of velocity decrease for both the fluid and dust phase. The presence of transverse magnetic field sets a resistive force, called Lorentz force, which opposes the velocity field. Therefore, as the values of $M$ increase so does the retarding force and hence the velocity decreases (Figs. 8-9). But Fig. 10 shows, axial component of velocity increases with increasing $M$, because of the fact that it is in same direction with the applied magnetic field. It is noticed from Figs. 11-12 
that an increase in magnetic parameter enhances the temperature as well as concentration profiles for both the phases. This is because of the resistive Lorentz force, which leads to enhancing the friction between adjacent fluid layers, as a result heat is produced and hence temperature and concentration increase.

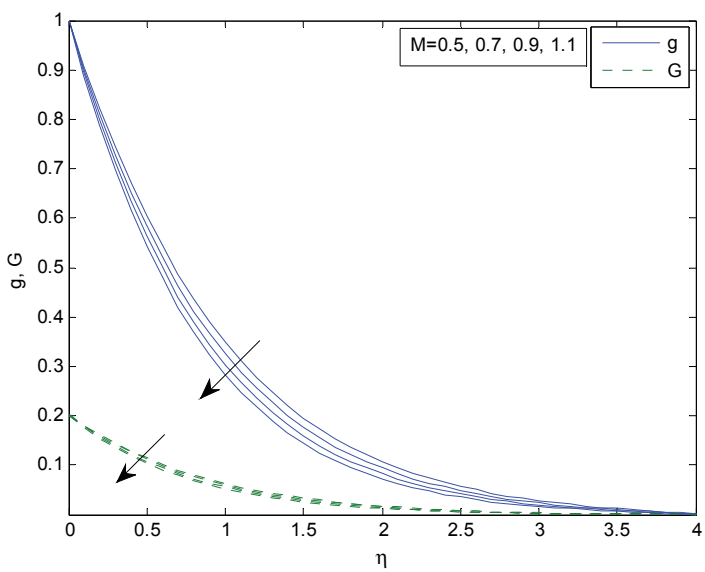

Fig. 9 Tangential velocity profile for different $M$

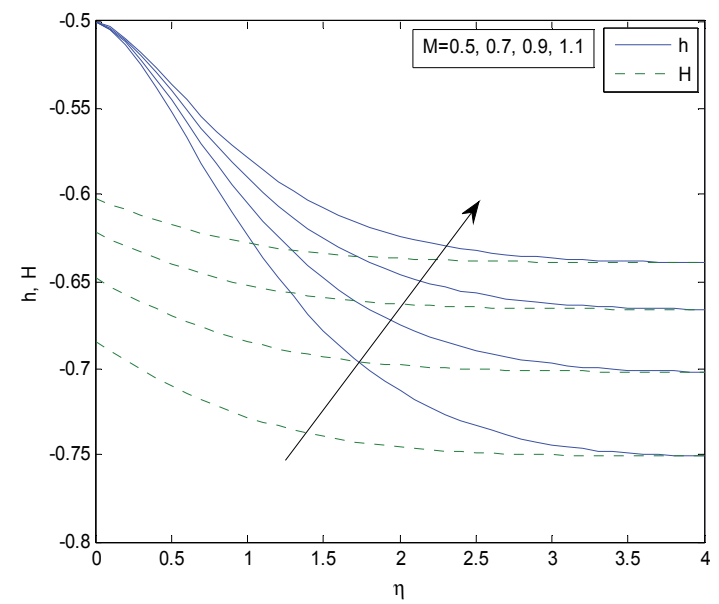

Fig. 10 Axial velocity profile for different $M$

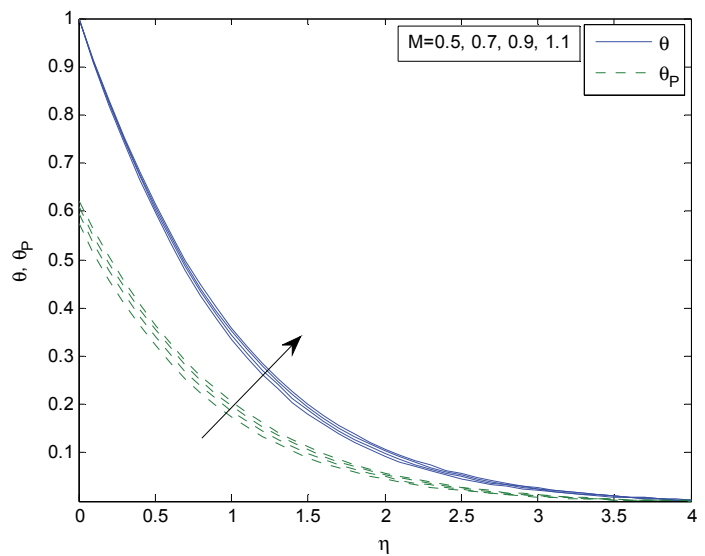

Fig. 11 Temperature profile for different $M$

Figure 13 depicts the temperature distribution for various values of radiation parameter $R_{d}$. From this figure it is seen that temperature of both the fluid and dust phase is maximum at the surface of the disk and asymptotically decreases to zero as $\eta$ increases.

Figures 14-18 elucidate the variation of velocity, temperature and concentration fields of fluid and dust phase with fluid particle interaction parameters. It is observed that, if $\beta_{v}, \beta_{T}$ and $\beta_{c}$ are increased then all the components of fluid phase velocity except axial component, temperature and concentration fields get decrease. But the velocity, temperature and concentration distributions for dust phase are significantly increased. It is also noticed from these graphs that, for very large values of $\beta_{v}, \beta_{T}$ and $\beta_{c}$ both the phases will be same.

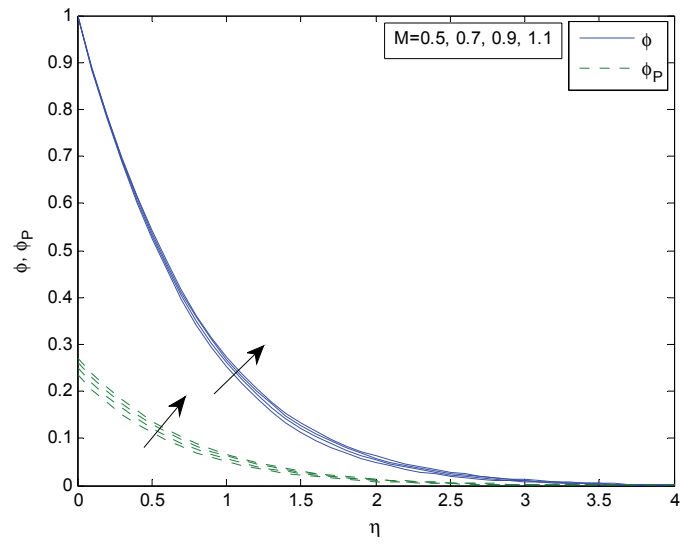

Fig. 12 Concentration profile for different $M$

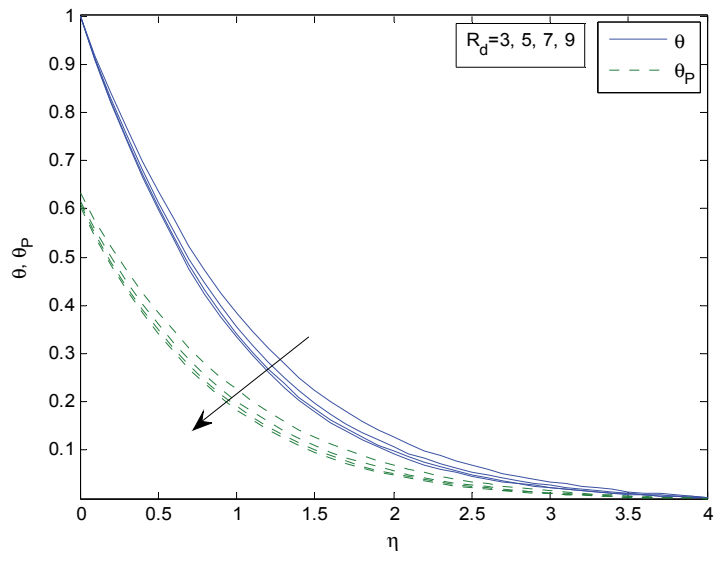

Fig. 13 Temperature profile for different $R_{d}$

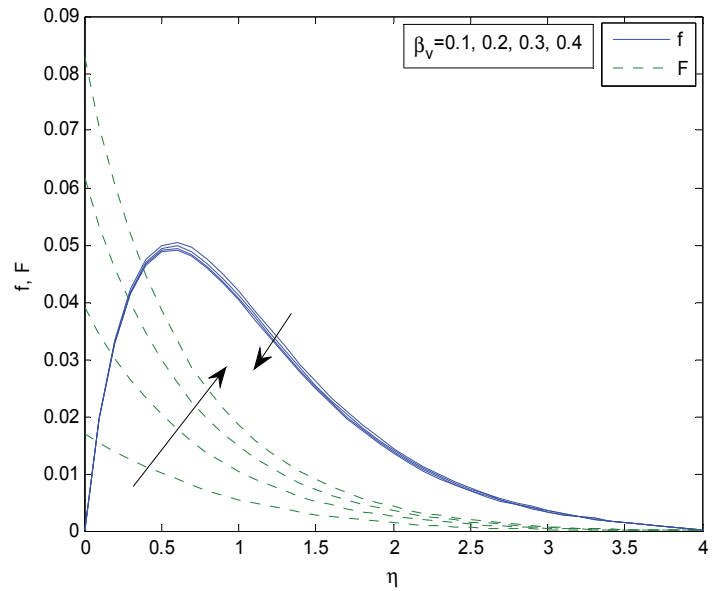

Fig. 14 Radial velocity profile for different $\beta_{v}$

The variations of velocity, temperature and concentration profiles for different values of Eckert number $(E c)$ are depicted in Figs. 19-23. It is found from Figs. 19-21 that, radial and tangential components of 
velocity increases with increasing $E c$ for both the fluid and dust phase. While the axial component of velocity profile significantly decreases with increasing Ec. By analyzing the graphs in Figs. 22-23, we have observed that an enhancement of $E c$ leads to increase the temperature and concentration distributions of both fluid and dust phase. This is because of the fact that the heat energy is stored in the fluid due to frictional heating.

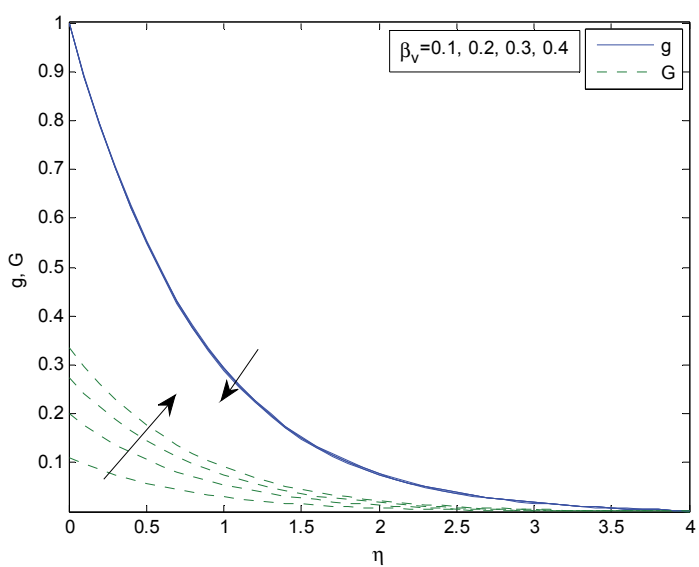

Fig. 15 Tangential velocity profile for different $\beta_{v}$

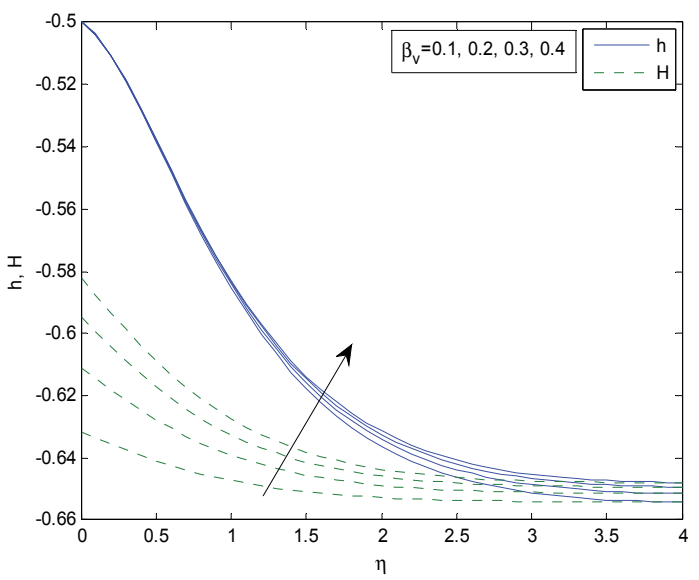

Fig. 16 Axial velocity profile for different $\beta_{v}$

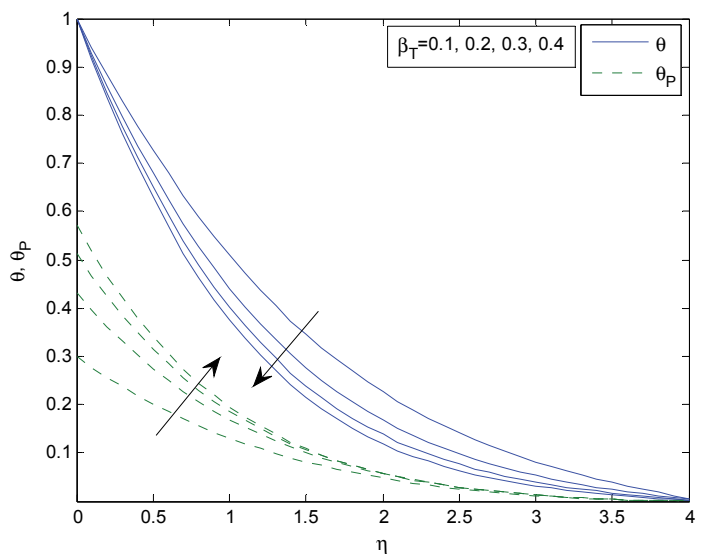

Fig. 17 Temperature profile for different $\beta_{T}$

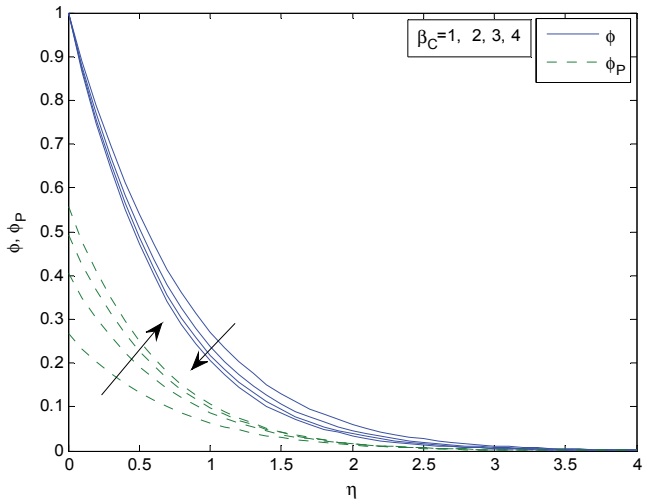

Fig. 18 Temperature profile for different $\beta_{c}$

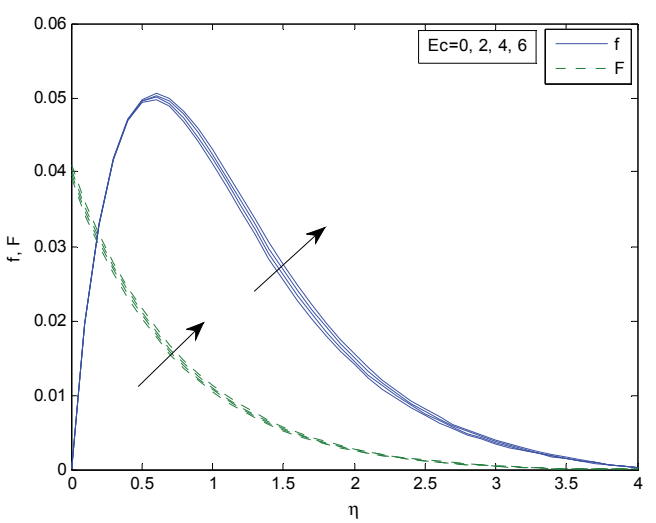

Fig. 19 Radial velocity profile for different $E c$

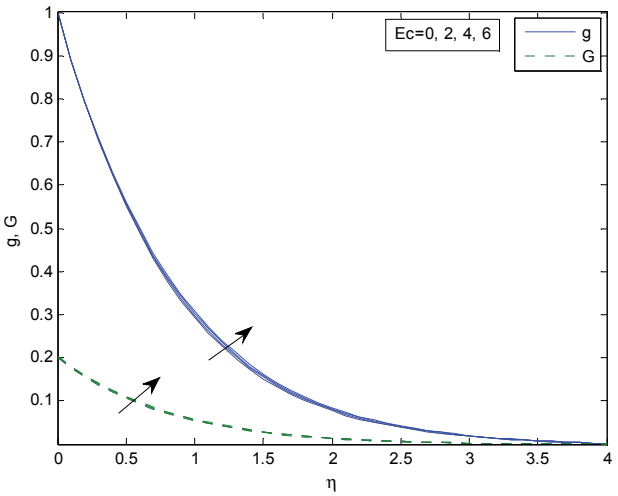

Fig. 20 Tangential velocity profile for different $E c$

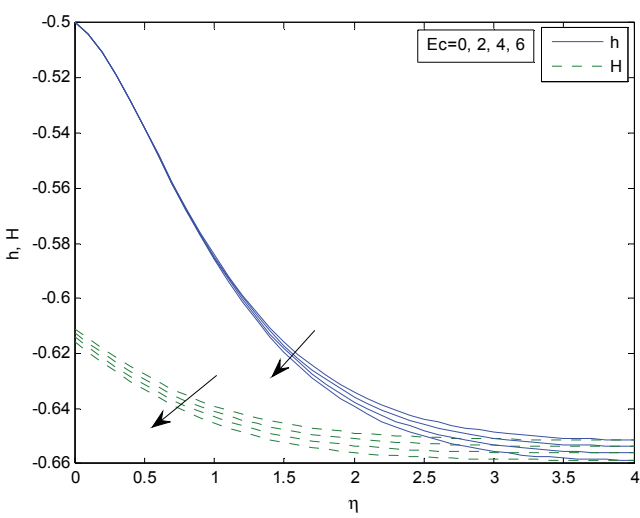

Fig. 21 Axial velocity profile for different $E c$ 


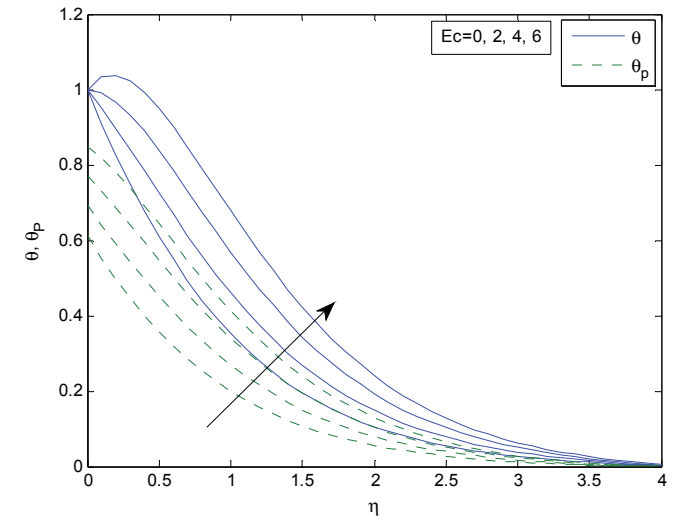

Fig. 22 Temperature profile for different $E c$

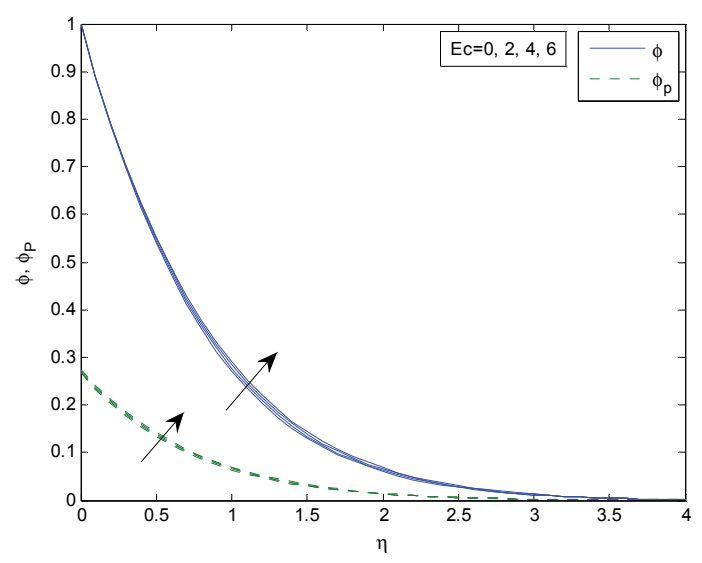

Fig. 23 Concentration profile for different $E c$

Table 1 Effects of $\theta_{r}, \theta_{c}, M, R_{d}$ and $E c$ on radial skin friction coefficient $\left(C_{f r}\right)$, tangential skin friction coefficient $\left(C_{f t}\right)$, Nusselt number $(N u)$ and Sherwood number(Sh)

\begin{tabular}{|c|c|c|c|c|c|c|c|c|}
\hline$\theta_{r}$ & $\theta_{c}$ & $M$ & $\mathrm{R}_{\mathrm{d}}$ & $\mathrm{Ec}$ & $C_{f t}$ & $C_{f r}$ & $\mathrm{Nu}$ & $S h$ \\
\hline 3 & \multirow{4}{*}{3} & \multirow{4}{*}{1} & \multirow{4}{*}{5} & \multirow{4}{*}{0.05} & -1.53146 & 0.730836 & 1.400648 & 1.525916 \\
\hline 5 & & & & & -1.4804 & 0.676131 & 1.393366 & 1.508651 \\
\hline 7 & & & & & -1.46009 & 0.655291 & 1.390404 & 1.501648 \\
\hline 9 & & & & & -1.44926 & 0.644093 & 1.388867 & 1.497852 \\
\hline \multirow{4}{*}{5} & 3 & \multirow{4}{*}{1} & \multirow{4}{*}{5} & \multirow{4}{*}{0.05} & -1.4804 & 0.676131 & 1.393366 & 1.508651 \\
\hline & 5 & & & & -1.47624 & 0.674312 & 1.322758 & 1.505433 \\
\hline & 7 & & & & -1.47463 & 0.673543 & 1.295707 & 1.504159 \\
\hline & 9 & & & & -1.47378 & 0.673113 & 1.281369 & 1.503475 \\
\hline \multirow{4}{*}{5} & \multirow{4}{*}{3} & 0.5 & \multirow{4}{*}{5} & \multirow{4}{*}{0.05} & -1.2214 & 0.820624 & 1.468169 & 1.555313 \\
\hline & & 0.7 & & & -1.32831 & 0.753691 & 1.433401 & 1.533553 \\
\hline & & 0.9 & & & -1.43083 & 0.699571 & 1.405288 & 1.51609 \\
\hline & & 1.1 & & & -1.52898 & 0.653797 & 1.382898 & 1.501801 \\
\hline \multirow{4}{*}{5} & \multirow{4}{*}{3} & \multirow{4}{*}{1} & 3 & \multirow{4}{*}{0.05} & -1.48309 & 0.67694 & 1.30425 & 1.510455 \\
\hline & & & 5 & & -1.4804 & 0.676131 & 1.393366 & 1.508651 \\
\hline & & & 7 & & -1.4791 & 0.675733 & 1.461799 & 1.507271 \\
\hline & & & 0 & & -1.47835 & 0.675498 & 1.461799 & 1.507271 \\
\hline \multirow{4}{*}{5} & \multirow{4}{*}{3} & \multirow{4}{*}{1} & \multirow{4}{*}{5} & 0 & -1.47995 & 0.675953 & 1.413352 & 1.508316 \\
\hline & & & & 2 & -1.49807 & 0.683085 & 0.600304 & 1.522102 \\
\hline & & & & 4 & -1.51717 & 0.690396 & -0.24263 & 1.536703 \\
\hline & & & & 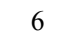 & -1.53729 & 0.697997 & -1.11872 & 1.552175 \\
\hline
\end{tabular}

The variation of skin friction coefficient, Nusselt number and Sherwood number for various flow governing parameters, viz. $\theta_{r}, \theta_{c}$, $M, R_{d}$ and $E c$ are shown in Table 1. From the table, it is noticed that

magnitude of tangential skin-friction coefficient decreases when viscosity variation parameter $\left(\theta_{r}\right)$, thermal conductivity variation parameter $\left(\theta_{c}\right)$ and radiation parameter $\left(R_{d}\right)$ increase; however, it increases as the values of Eckert number increase. Also, this table indicates radial skin-friction coefficient decreases for increasing values of $\theta_{r}, \theta_{c}$ and $R_{d}$; while it increases with $E c$ increases. The Nusselt number i.e., rate of heat transfer at the disk decreases with an increase in $\theta_{r}, \theta_{c}$ and $E c$; but we have observed a slight increase with $R_{d}$. The Sherwood number i.e., the rate of mass transfer decreases for an enhancement of the parameters $\theta_{r}, \theta_{c}$ and $R_{d}$, whereas it increases with Eckert number $E c$. All the physical quantities of interest skinfriction coefficient, Nusselt number and Sherwood number decrease as the value of magnetic parameter $M$ increases.

\section{CONCLUSIONS}

In this paper, an analysis is performed on the effects of viscosity variation parameter, thermal conductivity variation parameter, magnetic parameter, fluid particle interaction parameters, radiation parameter and Eckert number on a conducting dusty fluid flow due to a rotating disk with the influence of magnetic field taking into account.

From the analysis following conclusions were made:

- It is clear that the effects of temperature on viscosity and thermal conductivity are very significant.

- The axial component of the velocity increases with increasing values of viscosity variation parameter and magnetic parameter.

- The effect of the viscosity variation parameter, magnetic parameter and Eckert number is seen to increase the temperature profiles of both the fluid and dust phases.

- An enhancement of radiation parameter has a tendency to decrease the temperature distribution of both the fluid and dust phases.

- $\quad$ An increase in the value of viscosity variation parameter leads to decrease the concentration profile of both the fluid and dust phases; while concentration increases for increasing values of the magnetic parameter and Eckert number.

- It is observed that velocity and temperature profiles of both the fluid and dust phase will be same for very large values of fluid particle interaction parameter.

- The magnitude of skin friction coefficient predicted to be decreased with increase in the value of viscosity variation, thermal conductivity variation and radiation parameters; whereas skin friction coefficient increases with Eckert number increases.

- It is noticed that rate of heat and mass transfer decreases with viscosity variation, thermal conductivity variation and magnetic parameters increase.

\section{ACKNOWLEDGEMENTS}

Authors are thankful to the University Grants Commission, New Delhi, India, for the financial support under the scheme of National Fellowship for Students of Other Backward Classes (NFOBC).

\section{NOMENCLATURE}

$B_{0}$

C

$\mathrm{C}_{\mathrm{p}}$

$\mathrm{c}_{\mathrm{m}}$

$\mathrm{c}_{\mathrm{p}}$ axial component of magnetic field strength

concentration of the fluid

concentration of dust phase

specific heat of dust particles at constant pressure

specific heat of fluid at constant pressure 


\begin{tabular}{|c|c|}
\hline$D m$ & mass diffusivity \\
\hline$f, F$ & $\begin{array}{l}\text { dimensionless radial component of fluid and dust phase } \\
\text { velocity, respectively }\end{array}$ \\
\hline$g, G$ & $\begin{array}{l}\text { dimensionless tangential component of fluid and dust phase } \\
\text { velocity, respectively }\end{array}$ \\
\hline$h, H$ & $\begin{array}{l}\text { dimensionless axial component of fluid and dust phase } \\
\text { velocity, respectively }\end{array}$ \\
\hline$K$ & Stokes' resistance (drag co-efficient) \\
\hline$l$ & mass concentration \\
\hline $\mathrm{m}$ & mass of a dust particle \\
\hline$N$ & number density of the particle phase \\
\hline$p$ & pressure \\
\hline $\mathrm{P}$ & dimensionless pressure \\
\hline$T$ & temperature of the fluid inside the boundary layer \\
\hline$T_{p}$ & temperature of the dust phase inside the boundary layer \\
\hline$T_{\infty}$ & temperature of the ambient fluid \\
\hline \multicolumn{2}{|c|}{ Greek Symbols } \\
\hline$\theta, \theta_{p}$ & dimensionless temperature of fluid and dust phase, \\
\hline$\lambda$ & $\begin{array}{l}\text { respectively } \\
\text { thermal conductivity }\end{array}$ \\
\hline$\lambda_{\infty}$ & thermal conductivity of the ambient fluid \\
\hline$\mu$ & dynamic viscosity \\
\hline$\mu_{\infty}$ & dynamic viscosity of the ambient fluid \\
\hline$\phi, \phi_{p}$ & dimensionless species concentration of fluid and dust \\
\hline$\rho$ & $\begin{array}{l}\text { phase, respectively } \\
\text { density of the fluid }\left(\mathrm{kg} / \mathrm{m}^{3}\right)\end{array}$ \\
\hline$\rho_{p}$ & density of the dust phase $\left(\mathrm{kg} / \mathrm{m}^{3}\right)$ \\
\hline$\sigma$ & electrical conductivity \\
\hline$\tau_{v}$ & $\begin{array}{l}\text { relaxation time of the dust particles i.e., the time required } \\
\text { by a dust particle to adjust its velocity to the fluid }\end{array}$ \\
\hline$\tau_{T}$ & $\begin{array}{l}\text { thermal equilibrium time and is the time required by the } \\
\text { dust cloud to adjust its temperature relative to the fluid }\end{array}$ \\
\hline$\tau_{C}$ & $\begin{array}{l}\text { solutal equilibrium time i.e., the time required by the } \\
\text { dust particle to adjust its concentration relative to the fluid }\end{array}$ \\
\hline$v_{\infty}$ & kinematic viscosity of the fluid in the free stream \\
\hline$\omega$ & constant density ratio \\
\hline
\end{tabular}

\section{REFERENCES}

Attia, H. A., 2006, "Unsteady MHD Couette Flow and Heat Transfer of Dusty Fluid with Variable Physical Properties," Applied Mathematics and Computation, 177(1), 308-318.

http://dx.doi.org/10.1016/j.amc.2005.11.010

Choudhury, M., and Hazarika, G. C., 2013, "The Effects of Variable Viscosity and Thermal Conductivity on MHD Oscillatory Free Convective Flow past a Vertical plate in Slip Flow Regime with Variable Suction and Periodic plate Temperature," J. Appl. Fluid Mech, 6(2), 277-283.

Debnath, L., Ray, S.C., and Chatterjee, A.K., 1979, "Effects of Hall Current on Unsteady Hydromagnetic Flow past a Porous Plate in a Rotating Fluid System," Zeitschrift für Angewandte Mathematik und Mechanik, 59(9), 469-471.

http://dx.doi.org/10.1002/zamm.19790590910

Devi, S. P. A., and Devi, R. U., 2011, "On Hydromagnetic Flow due to a Rotating Disk with Radiation Effects," Nonlinear Analysis: Modelling and Control, 16(1), 17-29.

https://www.mii.lt/na/issues/NA 1601/NA16102.pdf
Datta, N., and Mishra, S. K., 1982, "Boundary Layer Flow of a Dusty Fluid over a Semi-infinite Flat Plate," ActaMechanica, 42(1), 71-83. http://dx.doi.org/10.1007/BF01176514

Gireesha, B. J., Chamkha, A. J., Manjunatha, S., and Bagewadi, C. S. 2013, "Mixed Convective Flow of a Dusty Fluid over a Vertical Stretching sheet with Non-uniform Heat Source/Sink and Radiation," Int. J. Numer Methods Heat Fluid Flow, 23(4), 598-612. http://dx.doi.org/10.1108/09615531311323764

Gireesha, B. J., Ramesh, G. K., Abel Subhas, M., and Bagewadi, C. S., 2011, "Boundary Layer Flow and Heat Transfer of a Dusty Fluid Flow over a Stretching Sheet with non-uniform Heat Source/Sink," Int. J. Multiphase Flow, 37(8), 977-982.

http://dx.doi.org/10.1016/j.ijmultiphaseflow.2011.03.014

Gireesha, B. J., Roopa, G. S., and Bagewadi, C. S., 2012, "Effect of Viscous Dissipation and Heat Source on Flow and Heat Transfer of a Dusty Fluid over Unsteady Stretching sheet," Appl. Math. Mech. Engl Ed., 33(8), 1001-1014. http://dx.doi.org/10.1007/s10483-012-1601-9

Gupta, P. K., and Gupta, S. C., 1976, "Flow of a Dusty Gas through a Channel with Arbitrary Time varying Pressure Gradient," Journal of Appl Math and Phys, 27, 119.

Hazarika, G. C., and Konch, J., 2016, "Effects of Variable Viscosity and Thermal Conductivity on Magnetohydrodynamic Free Convection Dusty Fluid along a Vertical Porous plate with Heat Generation," Turkish Journal of Physics, 40(1), 52-68. http://dx.doi.org/10.3906/ z-1509-14

Herwig, H., and Wicken, G., 1986, "The Effect of Variable Properties on Laminar Boundary layer Flow," Warme-und Stoffubertragung, 20, 47-57.

Kanch, A. K., and Jana, R. N., 1999, "Hall Effects on Unsteady Hydromagnetic Flow past an Eccentrically Rotating Porous Disk in a Rotating Fluid," Czech. J. Phys., 49(2), 205-215.

https://doi.org/10.1023/A:1022802011874

Khidir, A. A., 2013, "Viscous Dissipation, Ohmic Heating and Radiation Effects on MHD Flow past a Rotating Disk embedded in a Porous Medium with Variable properties," Arab J Math, 2, 263-277 https://doi.org/10.1007/s40065-013-0072-3

Konch, J., and Hazarika, G. C., 2016, "Effects of Variable Viscosity and Thermal Conductivity on MHD Free Convection Flow of Dusty Fluid along a Vertical Stretching sheet with Heat Generation," International Research Journal of Engineering and Technology, 3(2), 1029-1038.

https://www.irjet.net/archives/V3/i2/IRJET-V3I2184.pdf

Kuppalapalle, V., Vinayaka, P. K., and Chiu-On, N., 2013, “The Effect of Variable Viscosity on the Flow and Heat Transfer of a Viscous Agwater and Cu-water Nanofluids," Journal of Hydrodynamics, 25(1), 19.

https://doi.org/10.1016/S1001-6058(13)60332-7

Lai, F. C., and Kulacki, F. A., 1991, "The Effect of Variable Viscosity on Convective Heat and Mass Transfer along a Vertical Surface in Saturated Porous Media," Int. J. of Heat and Mass Transfer, 33, 10281031.

Ling, J. X., and Dybbs, A., 1987, "Forced Convection over a Flat Plate submersed in a Porous Medium: Variable Viscosity case," ASME Winter Annual Meeting, Boston, Massachusetts, 13-18.

Mallikarjuna, B., Rashad, A. M., Chamkha, A. J., and Hariprasad Raju, S., 2016, "Chemical reaction Effects on MHD Convective Heat and Mass Transfer Flow past a Rotating Vertical Cone embedded in a Variable Porosity Regime," Afr. Mat., 27, 645-665.

https://doi.org/10.1007/s13370-015-0372-1 
Manjunatha, P. T., Gireesha, B. J., and Prasannakumara, B. C., 2015, "Effect of Radiation on Flow and Heat Transfer of MHD Dusty Fluid over a Stretching Cylinder Embedded in a Porous Medium in presence of Heat Source," Int. J. Appl. Comput. Math. http://dx.doi.org/10.1007/s40819-015-0107-x.

Michael, D. H., and Miller, D. A., 1966, "Plane Parallel Flow of a Dusty Gas", Mathematika, 13, 97-109. https://doi.org/10.1112/S0025579300004289

Millsaps, K., and Pohlhausen, K., 1952, "Heat Transfer by Laminar Flow from a Rotating Disk," J. Aeronaut. Sci., 19, 120-126.

Nayfeh, A. H., 1966, "Oscillating Two-Phase Flow through a Rigid Pipe,” AIAAJ, 4(10), 1868-1870.

http://dx.doi.org/10.2514/3.3804

Necati Özisik, M., 1973, "Radiative Transfer," John Wiley \& Sons, New York.

Palani, G., and Kim, Kwang-Yong, 2010, "Free Convection of a DustyGas Flow along a Semi-infinite Vertical Cylinder," Int. J. Numer. Meth. Fluids, 63(4), 517-532. http://dx.doi.org/10.1002/fld.2082

Prasada Rao, D. R. V., and Krishna, D. V., 1981, "Hall Effect on Unsteady Hydromagnetic Flow," Indian J. Pure. Appl. Math., 12(2), 270-276.

http://insa.nic.in/writereaddata/UpLoadedFiles/IJPAM/20005a81 270.p df
Ramamurthy, V., 1990, "Free Convection Effects on the Stokes Problem for an Infinite Vertical Plate in a Dusty Fluid," J. Math. Phys. Sci., 24, 297-312.

Saffman, P. G., 1962, "On the Stability of Laminar Flow of a Dusty Gas," Journal of Fluid Mechanics, 13(1), 120-128. https://doi.org/10.1017/S0022112062000555

Sibanda, P., and Makinde, O.D., 2010, "On Steady MHD Flow and Heat Transfer past a Rotating Disk in a Porous Medium with Ohmic Heating and Viscous Dissipation," Int. J. Numer. Methods Heat Fluid Flow, 20(3), 269-285.

http://dx.doi.org/10.1108/09615531011024039

Sutton, G. W., and Sherman, A., 1965, "Engineering Magnetohydrodynamics," New York: McGraw-Hill.

Tiwari, R., and Singh, K., 1983, "Effect of Hall Current on Unsteady Hydromagnetic Boundary Layer in Rotating Dusty Fluid," Indian. J. Pure Appl. Math., 14(1), 159-165.

http://insa.nic.in/writereaddata/UpLoadedFiles/IJPAM/20005a7c 159.p df

Turkyilmazoglu, M., 2009, "Purely Analytic Solutions of the Compressible Boundary Layer Flow due to a Porous Rotating Disk with Heat Transfer," Phys. of Fluids, 21(10), 106104. http://dx.doi.org/10.1063/1.3249752

Turkyilmazoglu, M., 2010, "The MHD Boundary Layer Flow due to a Rough Rotating Disk," ZAMM Z. Angew. Math. Mech., 90(1), 72-82. http://dx.doi.org/10.1002/zamm.200900259 